\title{
AVALIAÇÃO DA QUALIDADE E ACEITAÇÃO DE FISHBURGUERS DE TILÁPIA PROCESSADOS COM FARINHA DAS CASCAS DE UMBU E DE UMBU-CAJÁ COMO PROVÁVEL ANTIOXIDANTE NATURAL
}

\author{
QUALITY ASSESSMENT AND ACCEPTANCE OF \\ FISHBURGUERS OF TILAPIA PROCESSED WITH FLOUR OF \\ BARKS OF UMBU AND UMBU-CAJA AS PROBABLE NATURAL \\ ANTIOXIDANT
}

\author{
Graciete de Souza Silva ${ }^{1}$; Marcelo Iran de Souza Coelho ${ }^{2}$; Graciene de Souza Silva ${ }^{3}$ Alessandra Sousa \\ Cordeiro de $\mathrm{Sá}^{4}$ \\ ${ }^{1,2,3,4}$ Instituto Federal de Educação, Ciência e Tecnologia do Sertão Pernambucano - IF SERTÃO PE - \\ Petrolina - Brasil graci.ete@hotmail.com.br
}

\begin{abstract}
Resumo
Produtos elaborados com a carne de peixe, assim como outros derivados cárneos, apresentam estabilidade lipídica baixa devido à liberação de enzimas na carne desintegrada, incorporação de oxigênio e a presença de ácidos graxos insaturados, o que interfere na qualidade e durabilidade destes produtos. A utilização de antioxidantes tem sido considerada uma técnica eficiente para retardar ou mesmo inibir a oxidação de lipídios nos alimentos. Não obstante, muitos estudos têm comprovado que alguns antioxidantes sintéticos podem favorecer efeitos mutagênicos $e$ carcinogênicos, entre outros, e por isso a busca por antioxidantes naturais que possam substituir ou reduzir o uso dos sintéticos tem sido impulsionada. Pesquisas recentes demonstram que compostos existentes nas cascas de frutos do umbuzeiro e espécies afins, como o ácido ascórbico, carotenoides e compostos fenólicos, apresentam expressivo potencial antioxidante. Estas constatações motivaram o desenvolvimento deste trabalho que teve como objetivo avaliar a aceitação de formulações de fishburgers de tilápia (Oreochromis niloticus), contendo farinha de cascas de umbu (Spondias tuberosa) e de umbu-cajá (Spondias sp), como antioxidante natural. Foram realizadas análises físico-químicas das farinhas, testes afetivos de aceitabilidade dos produtos prontos e análise estatística dos dados das variáveis. Os resultados comprovaram à adequação do fishburguer formulado aos padrões de identidade e qualidade exigidos e revelaram que a adição das farinhas neste produto, não interferiu na aceitação pelos provadores.
\end{abstract}

Palavras-chave: formulações; peixe; sensorial . 


\section{Introdução}

As transformações do consumo de pescado, aliadas ao hábito alimentar da população, têm sido responsáveis pela crescente demanda por peixes no Brasil. Em expansão, várias áreas relacionadas à produção de pescado são necessárias para o desenvolvimento sustentável de toda cadeia produtiva. No entanto, a exploração e uso do pescado no país ainda não alcançam os benefícios nutricionais e econômicos que deles se espera.

De acordo com afirmações de Parmigiani e Torres (2005), em 2004 o Brasil já apresentava um dos mais baixos índices de consumo de pescado, bem abaixo da média mundial. Este fato pode ser explicado, não só por problemas na distribuição e comercialização, como também muitas vezes pela falta do hábito de consumo, gerados em parte pela ausência de praticidade no preparo.

O recém-criado Ministério da Pesca e Aquicultura (MPA) do governo brasileiro pretende aumentar o consumo de pescado dos $7 \mathrm{Kg}$ por habitante/ano para $9 \mathrm{~kg}$ por habitante ano, e para alcançar esta meta prevê um aumento de $50 \%$ da produção proveniente da aquicultura (MPA, 2011). Para isso, acredita-se que o incentivo ao processamento de peixes e desenvolvimento de novos produtos poderá favorecer este aumento.

Dentre as espécies de peixe utilizadas para o processamento, pode ser citada a tilápia-do-nilo (Oreochromis niloticus), considerada atualmente a espécie mais cultivada na piscicultura brasileira e uma das mais importantes da atividade no mundo (FAO, 2007). Uma espécie exótica bastante encontrada na região do Vale do São Francisco e também muito utilizada na produção de linguiças, almôndegas, espetinhos, fishburgers, entre outros produtos.

Com relação aos produtos elaborados com a carne de peixe, estes possuem estabilidade lipídica baixa devido à liberação de enzimas na carne desintegrada, incorporação de oxigênio e presença de ácidos graxos insaturados (OETTERER et al., 2006). Deste modo, a utilização de técnicas no retardo da oxidação lipídica, como o uso de antioxidantes pode ser uma alternativa válida para prolongar a vida de prateleira, já que as alterações provocadas devido à oxidação são relevantes não só em relação à qualidade dos alimentos, mas também, sob o enfoque econômico (BOCHI, 2007).

Pesquisas recentes vêm revelando que as cascas e as sementes de certos frutos exibem teores relevantes de fitoquímicos bioativos cuja atividade antioxidante é mais elevada do que a polpa. (JORGE, 2009; LUZIA e JORGE, 2010; MELO, 2010; SOARES, 2008). Frutos do gênero Spondias, como o umbu (Spondias tuberosa) e o umbu-cajá (Spondias sp), cultura nativa da região Nordeste do país, por exemplo, estão sendo alvo de muitas pesquisas científicas neste sentido.

Estudos revelam que compostos existentes na polpa ou mesmo em resíduos do umbu e umbu-cajá, como: ácido ascórbico, carotenoides e compostos fenólicos, apresentam expressivo potencial antioxidante (MELO, 2010; MOREIRA, 2011). Os fitoquímicos existentes na composição 
destes frutos atuam retardando a velocidade da reação de oxidação e podem ser vistos como um material promissor para a extração de compostos bioativos, com possibilidade de aplicação em alimentos como antioxidante natural, inclusive em produtos derivados de peixe.

Neste contexto, o presente trabalho teve como objetivo avaliar a qualidade e aceitação de formulações de Fishburguer de tilápia (Oreochromis niloticus) adicionadas de farinhas de umbu e umbu-cajá como provável fonte de antioxidante natural.

\section{Material e Métodos}

Processamento das Farinhas de Cascas do Umbu-cajá (Spondias sp) e do Umbu (Spondias tuberosa).

Os frutos maduros e semi maduros de umbu (Spondias tuberosa) e umbu-cajá (Spondias sp) foram adquiridos em uma feira livre do município de Campo Formoso-BA (20 kg de cada fruta) e transportados para a Agroindústria do Instituto Federal do Sertão Pernambucano, no Campus Petrolina Zona Rural, onde foram elaboradas a Farinhas da Casca de Umbu (FCU) e Farinha da Casca de Umbu-cajá (FCUC), conforme os procedimentos descritos no fluxograma ( Figura 1.)

Figura 1 - Fluxograma de processamento das farinhas

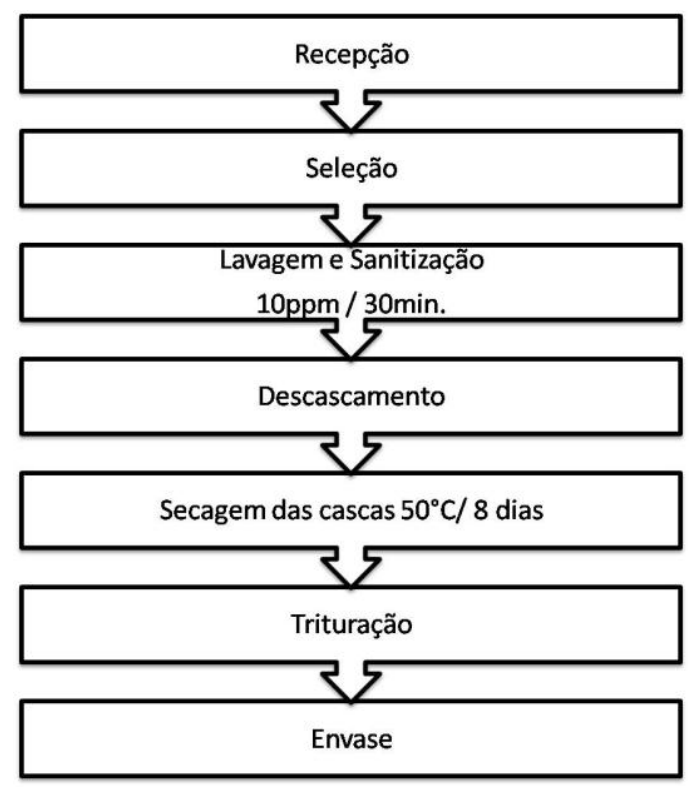

A sanitização dos frutos foi realizada submetendo-os à lavagem em água corrente e posteriormente à imersão em recipientes de polietileno contendo solução de hipoclorito de sódio comercial a $10 \mathrm{ppm}$, durante 20 minutos (Figura 2). Após isso, foram descascados manualmente para obtenção das cascas, as quais foram colocadas em estufa com circulação forçada de $\operatorname{ar}\left(50^{\circ} \mathrm{C}\right)$, 
durante oito dias, quando atingiram umidade inferior a 10\%. A polpa fresca obtida foi utilizada no processamento de geleia.

As cascas secas foram trituradas em liquidificador e em seguida passadas em um moinho para obtenção de uma farinha fina e de granulometria uniforme; A FCU e FCUC foram embaladas a vácuo em embalagens de polietileno para posterior análise físico-química.

Figura 2 - Sanitização dos frutos: umbu (spondias tuberosa) e umbu-cajá (spondias sp)

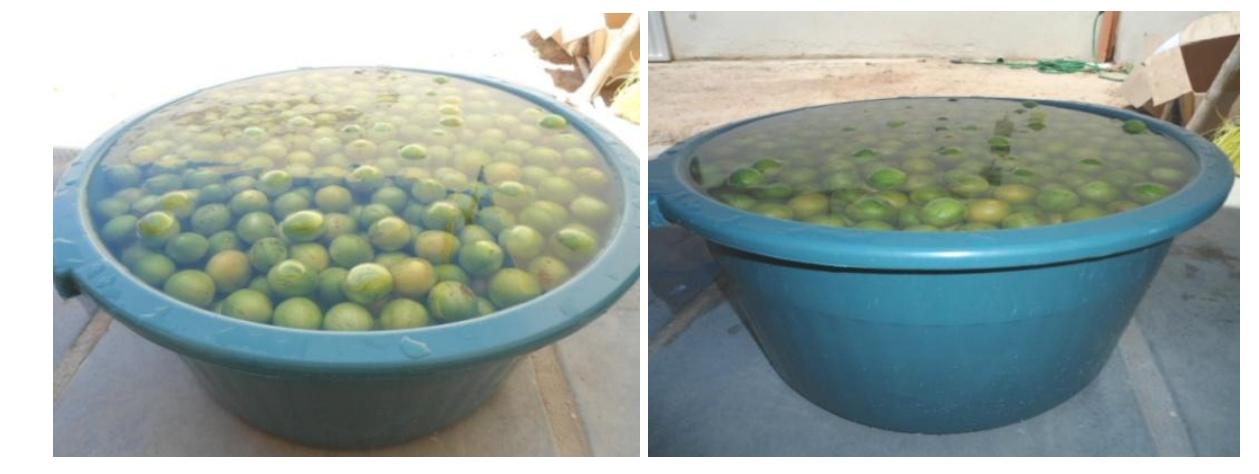

Fotos: Graciete Souza, 2012.

Caracterização Físico-química da Farinha de Cascas do Umbu (Spondias tuberosa) e Farinha de Cascas de Umbu-cajá (Spondias sp)

As farinhas obtidas foram conduzidas ao Laboratório de Pesquisa no Campus Petrolina para realização das análises físico-químicas, as quais foram realizadas conforme as Normas Analíticas do Instituto Adolfo Lutz (IAL, 1985).

O percentual de sólidos solúveis foi determinado com refratômetro de bancada, à temperatura de $25^{\circ} \mathrm{C}$. Quanto à umidade, esta foi avaliada por meio de estufa (Fanem) em temperatura de $105^{\circ} \mathrm{C}$, até estabilização do peso monitorado a cada quatro horas. Na etapa seguinte, as amostras secas na estufa, foram calcinadas em bico de bunsen, até completa carbonização e levadas a mufla aquecida a $550{ }^{\circ} \mathrm{C}$, até a obtenção das cinzas totais.

O teor de lipídios foi obtido a partir de extração direta em Soxhlet (IAL, 1985), tendo o hexano como solvente de extração. Da amostra seca e desengordurada contida no papel de filtro, foi realizada a análise de fibras de acordo com o método citado por Winton e Winton (1958) descrito por Henneberg, que simula in vitro o processo da digestão in vivo, de uma digestão em meio ácido, seguida por uma digestão em meio alcalino. A acidez titulável foi determinada com base na tilulação de neutralização de ácidos com solução padronizada de $\mathrm{NaOH} 0,1 \mathrm{~N}$ e com o uso de indicador de fenolftaleína. A leitura de $\mathrm{pH}$ foi feita com potenciômetro; Todos os dados foram obtidos em triplicatas e submetidos à análise estatística descritiva. 
Os peixes da espécie Tilápia do Nilo (oreochromis niloticus), utilizados no processamento dos fishburguers foram adquiridos no reservatório de piscicultura do Instituto Federal do Sertão Pernambucano no Campus Petrolina Zona Rural e conduzidos ao abatedouro deste mesmo Campus onde foram abatidos, descamados e eviscerados.

$\mathrm{Na}$ agroindústria, os peixes foram submetidos ao processo de obtenção do fishburguer, conforme procedimentos descritos no fluxograma (Figura 3).

Figura 3 - Fluxograma de produção de fishburger

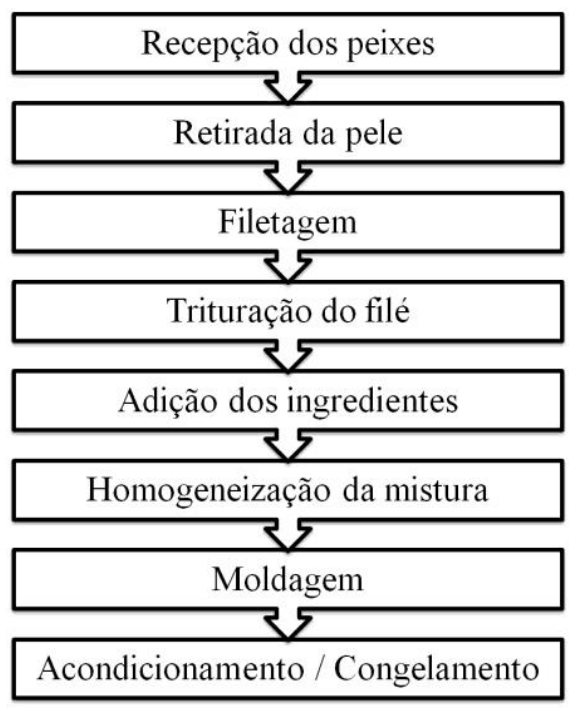

O filé obtido na filetagem foi triturado em moinho com discos de $5 \mathrm{~mm}$ e após a trituração elaborou-se as três formulações de fishburgers, conforme apresentado na tabela 1 .

Tabela 1 - Formulações de fishburguer de Tilápia

\begin{tabular}{l|c|c|c}
\hline & FSF & FFC & FFU \\
\hline Filéde Tilápia & $80,1 \%$ & $80 \%$ & $80 \%$ \\
Farinha detrigo & $2,8 \%$ & $2,9 \%$ & $2,9 \%$ \\
Cebola desidratada & $2,4 \%$ & $2,4 \%$ & $2,4 \%$ \\
Alho em pó & $0,8 \%$ & $0,8 \%$ & $0,8 \%$ \\
Proteina Texturizada de Soja & $2,3 \%$ & $2,3 \%$ & $2,3 \%$ \\
Água gelada & $6,7 \%$ & $6,5 \%$ & $6,5 \%$ \\
Oléo de semente de algodäo & $3,3 \%$ & $3,2 \%$ & $3,2 \%$ \\
Sal & $1,6 \%$ & $1,6 \%$ & $1,6 \%$ \\
Antioxidantenatural & $0,0 \%$ & $0,4 \%$ & $0,4 \%$ \\
\hline
\end{tabular}

FSF=Fishburger sem farinha; FFC=Fishburger com farinha de cajá;

FFU=Fishburger com farinha de umbu. 
Os fishburgers foram moldados com uma fôrma de plástico usada para moldar hambúrguer; os produtos foram mantidos congelados até o momento do preparo das amostras para a realização da análise sensorial (Figura 4).

Figura 4 - Fishburgers preparados para análise sensorial

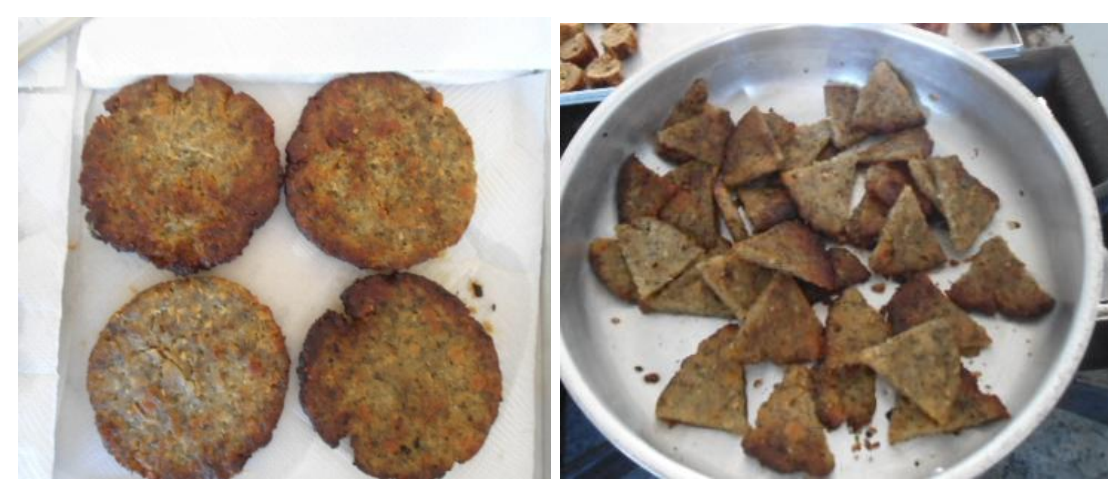

Fotos: Graciete Souza, 2012.

\section{Análise Sensorial e Físico-química}

As três formulações de fishburger, devidamente codificadas, foram submetidas à análise sensorial no Laboratório Experimental de Alimentos (LEA) do IF SERTÃO PE, com trinta provadores não treinados, de ambos os sexos, os quais receberam as três amostras do produto, cada um deles mais o formulário de avaliação e as devidas orientações para o preenchimento.

A análise foi realizada, com base em testes afetivos de aceitabilidade utilizando uma escala hedônica de sete pontos para os atributos: aparência, aroma, sabor e textura, incluindo ainda uma análise de preferência e intenção de compra (Figura 5).

Figura 5 - Formulário de Análise Sensorial

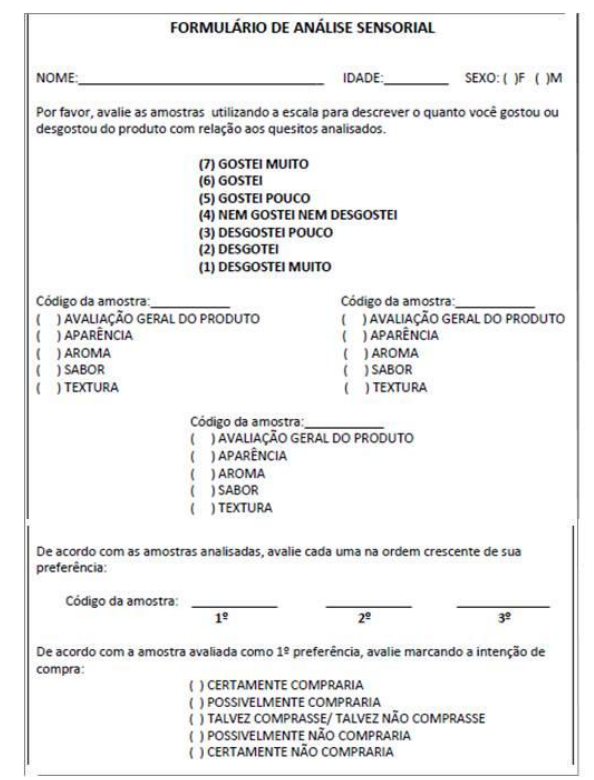


Durante a análise foi servido água em temperatura ambiente e biscoito de água e sal no intervalo entre cada formulação (Figura 6).

Figura 6- Análise Sensorial dos Fishburguers
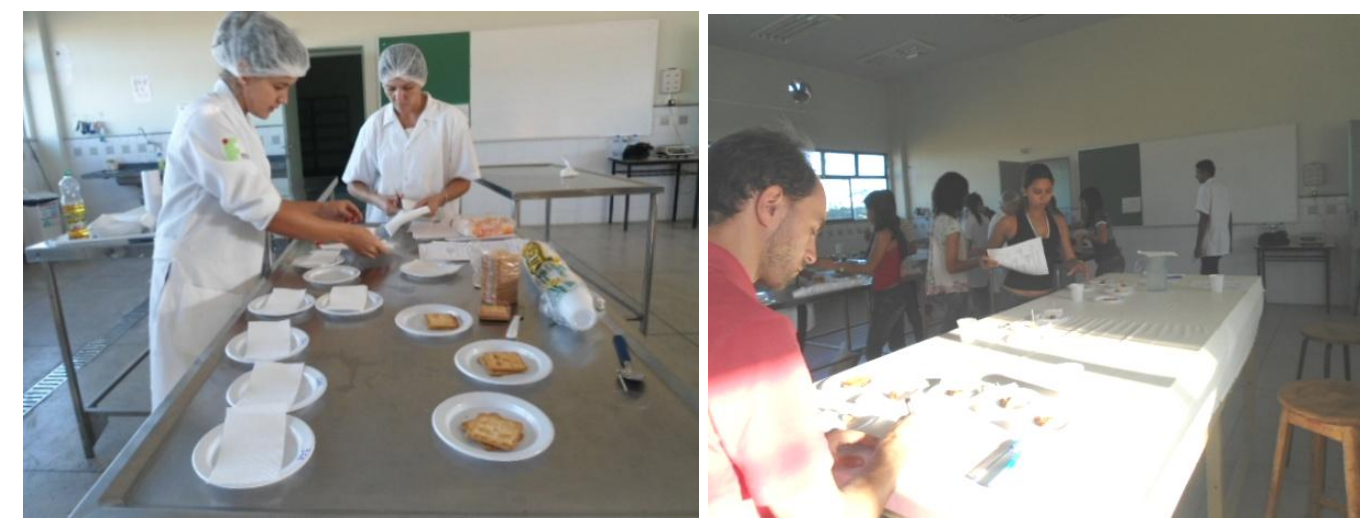

Fotos: Graciete Souza, 2012.

Os dados das variáveis foram submetidos à análise de variância - ANOVA e comparados entre si pelo teste de Tukey e Duncan a 5\% de significância e Teste de Correlação de Pearson, utilizando o software estatístico SPSS 14.0.

As formulações de fishburger preferidas em $1^{\circ}$ lugar pelos provadores na análise sensorial, foram caracterizadas sob parâmetros físico-químicos que incluíram: teor de umidade, cinzas totais, lipídeos e pH, realizadas conforme as Normas Analíticas do Instituto Adolfo Lutz (IAL,1985) e atividade de água (Aw) medida em um aparelho digital com câmara e sonda de Aw (Aw 43). As análises foram feitas em triplicatas em todos os parâmetros.

\section{Resultados e Discussão}

\section{Caracterização Físico-química das Farinhas}

As análises realizadas com as farinhas de cascas de umbu e de umbu-cajá revelaram a composição química destes produtos quanto ao teor de sólidos solúveis, umidade, cinzas, lipídios, acidez e pH, conforme apresentado nas tabelas 3 e 4. 
Tabela 2 - Características físico-químicas da Farinha de Cascas de Umbu (FCU)

\begin{tabular}{cccc}
\hline & Média & D.P.* & C.V.* \\
\hline Sólidos Solúveis Totais $\left({ }^{\circ}\right.$ Brix) & 58,35 & 1,39 & 2,38 \\
Umidade (\%) & 6,89 & 0,13 & 1,88 \\
Cinzas Totais (\%) & 2,89 & 1,17 & 4,77 \\
Lipídeos (\%) & 1,32 & 0,06 & 4,28 \\
Acidez Titulável (\%) & 0,98 & 0,22 & 22,18 \\
pH & 2,22 & 0,05 & 2,25 \\
\hline
\end{tabular}

*D.P.= Desvio Padrão / **C.V = Coeficiente de Variação

Tabela 3 - Características físico-químicas da Farinha de Cascas de Umbu Cajá (FCUC)

\begin{tabular}{cccc}
\hline & Média & D.P.* & C.V.* \\
\hline Sólidos Solúveis Totais $\left({ }^{\circ}\right.$ Brix $)$ & 36,08 & 0,05 & 1,13 \\
Umidade $(\%)$ & 4,70 & 0,03 & 0,57 \\
Cinzas Totais $(\%)$ & 3,70 & 0,04 & 0,97 \\
Lipídeos $(\%)$ & 2,77 & 0,36 & 13,12 \\
Acidez Titulável (\%) & 1,18 & 0,04 & 3,6 \\
$\mathbf{p H}$ & 1,97 & 0,01 & 1,59 \\
\hline
\end{tabular}

*D.P.= Desvio Padrão / **C.V = Coeficiente de Variação

Os resultados obtidos são relevantes para a pesquisa e demonstram teores significativos de minerais $(2,89 \%$ e $3,7 \%)$, sendo superiores aos valores reportados por alguns autores na polpa de frutos de espécies afins, como Santos et al. (2010), cujo valor encontrado foi de 0,99\% em cajás. O conteúdo de cinzas varia de $0,4 \%$ a $2,1 \%$ em frutas frescas e representam os minerais contidos nos alimentos que podem estar em grandes quantidades como o $\mathrm{K}+\mathrm{Na}+\mathrm{e} \mathrm{Ca}+$, e pequenas, como o ferro, Mn e Zn. (CECCHI, 2003). De acordo com Gondim, et al (2005), as cascas das frutas apresentam, em geral, teores de nutrientes maiores do que os das suas respectivas partes comestíveis.

Com relação ao percentual de umidade, os valores encontrados nas duas farinhas $(6,89 \%$ e 4,7\%), são inferiores aos de suas respectivas polpas, que fica em torno de 91,3\% (SANTOS, 2010), indicando que são produtos não perecíveis, já que a umidade está diretamente relacionada à perecibilidade dos alimentos. 
A acidez $(0,98 \%$ e 1,19\%) e o $\mathrm{pH}(2,22$ e 1,97) são fatores relevantes e também têm uma relação com a estabilidade dos produtos. Em se tratando de alimentos, valores de $\mathrm{pH}$ abaixo da neutralidade indicam maior estabilidade com relação a deterioração, os alimentos mais ácidos são mais estáveis, dificultando o desenvolvimento de microrganismos. E quanto aos teores de lipídios encontrados nas farinhas $(1,32 \%$ e $2,77 \%)$, estes foram valores baixos, demonstrando que são produtos estáveis a reações oxidativas.

\section{Análise Sensorial dos Fishburgers}

Com a realização da análise sensorial foi possível verificar a aceitação das formulações dos fishburgers (FSF=Fishburger sem farinha; $\mathrm{FFC}=$ Fishburger com farinha de cajá; FFU $=$ Fishburger com farinha de umbu) pelos provadores. Os resultados obtidos revelaram que não houve diferença significativa entre as médias das notas atribuídas para todos os atributos avaliados, de acordo com a análise de variância (ANOVA), conforme demonstrado nas tabelas 4, 5,6 e 7.

Tabela 4 - Resultados obtidos na avaliação da APARÊNCIA

\begin{tabular}{cccccc}
\hline \multirow{2}{*}{ TRATAMENTO } & MÉDIA & DP & CV(\%) & MIN & MAX \\
\hline FSF & $6,17 a$ & 1,15 & 18,63 & 5,74 & 6,60 \\
FFU & $6,07 a$ & 0,74 & 12,19 & 5,80 & 6,34 \\
FFC & $5,97 a$ & 0,85 & 14,23 & 5,65 & 6,30 \\
\hline
\end{tabular}

Médias acompanhadas de letras iguais indicam que não há diferença significativa pela ANOVA Teste F, considerando a margem de erro menor que 5\% / *Intervalo de Confiança

Tabela 5 - Resultados obtidos na avaliação do AROMA

\begin{tabular}{cccccc}
\hline \multirow{2}{*}{ TRATAMENTO } & \multirow{2}{*}{ MÉDIA } & DP & CV(\%) & \multicolumn{2}{c}{ IC* $(95 \%)$} \\
\hline FSF & $6,00 \mathrm{a}$ & 0,87 & 14,50 & 5,67 & MAX \\
FFU & $6,20 \mathrm{a}$ & 1,09 & 17,58 & 5,80 & 6,61 \\
FFC & $6,03 \mathrm{a}$ & 0,96 & 15,92 & 5,67 & 6,40 \\
\hline
\end{tabular}

Médias acompanhadas de letras iguais indicam que não há diferença significativa pela ANOVA - Teste F, considerando a margem de erro menor que 5\% / *Intervalo de Confiança 
Tabela 6 - Resultados obtidos na avaliação do SABOR

\begin{tabular}{cccccc}
\hline \multirow{2}{*}{ TRATAMENTO } & \multirow{2}{*}{ MÉDIA } & \multirow{2}{*}{ DP } & \multirow{2}{*}{ CV(\%) } & \multicolumn{2}{c}{ IC $^{*}(95 \%)$} \\
\hline FSF & $6,30 a$ & 0,84 & 13,33 & 5,99 & 6,61 \\
FFU & $6,17 a$ & 0,75 & 12,15 & 5,90 & 6,45 \\
FFC & $6,33 a$ & 1,15 & 18,17 & 5,90 & 6,76 \\
\hline
\end{tabular}

Médias acompanhadas de letras iguais indicam que não há diferença significativa pela ANOVA Teste F, considerando a margem de erro menor que 5\% / * Intervalo de Confiança

Tabela 7 - Resultados obtidos na avaliação do TEXTURA

\begin{tabular}{cccccc}
\hline \multirow{2}{*}{ TRATAMENTO } & \multirow{2}{*}{ MÉDIA } & \multirow{2}{*}{ DP } & \multirow{2}{*}{ CV(\%) } & MIN & MAX \\
\hline FSF & $6,03 a$ & 1,00 & 16,58 & 5,66 & 6,41 \\
FFU & $6,20 a$ & 0,80 & 12,90 & 5,90 & 6,50 \\
FFC & $6,30 a$ &, 092 & 14,60 & 5,96 & 6,64 \\
\hline
\end{tabular}

Médias acompanhadas de letras iguais indicam que não há diferença significativa pela ANOVA - Teste F, considerando a margem de erro menor que $5 \% / *$ Intervalo de Confiança

Posteriormente à análise de variância foi realizado o teste de comparação das médias Tukey e Duncan, os quais reforçaram os primeiros resultados obtidos, constatando que os provadores, de maneira geral, julgaram de forma semelhante as três formulações de fishburgers, o que pode ser visto como um fator positivo na tentativa de incluir estas farinhas como antioxidante natural nos produtos elaborados, uma vez que não houve diferenças significativas entre as formulações sem farinha e as demais.

É possível verificar ainda que em uma escala de 1 a 7 pontos, as médias das notas atribuídas para as formulações de fishburgers encontram-se em torno de " 6 " indicando que os provadores gostaram das amostras.

Os resultados alcançados com a análise de preferência revelaram que a maior parte dos provadores (40\%) escolheu a formulação de fishburger adicionada de farinha de cascas de umbucajá como preferida em $1^{\circ}$ lugar e entre estes avaliadores $50 \%$ indicaram que "certamente compraria" a amostra caso fosse lançada no mercado (Figura 8). 
Figura 8 - Resultado do Teste de Preferência e Intenção de Compra dos Fishburgers

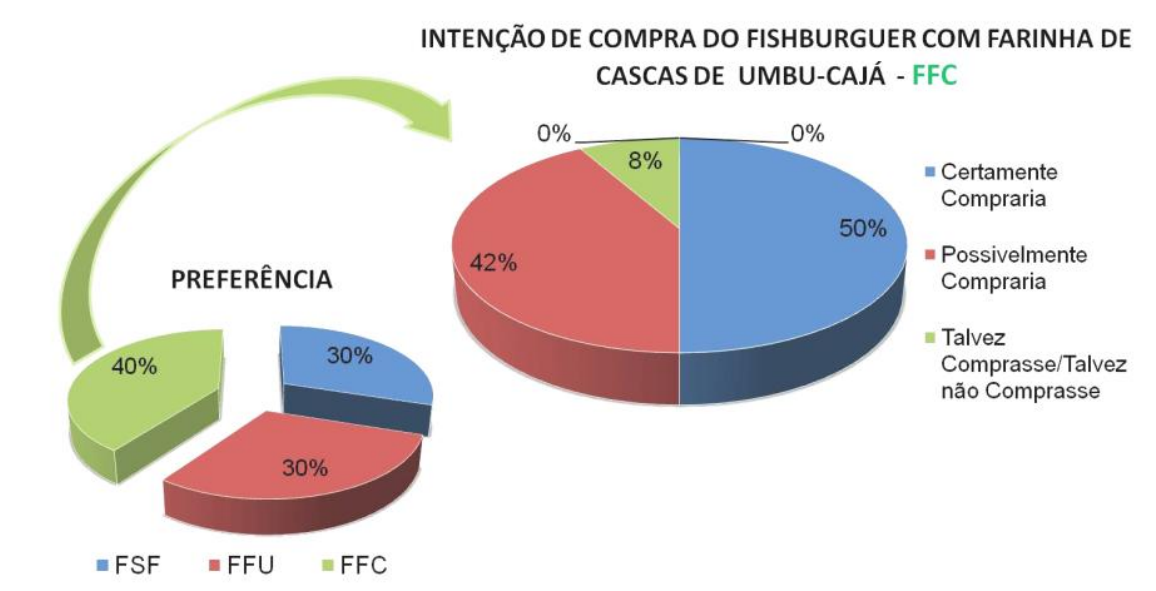

$\mathrm{FSF}=$ fishburger sem farinha; $\mathrm{FFU}=$ fishburger com farinha de cascas de umbu; $\mathrm{FFC}=$ fishburger $\mathrm{com}$ farinha de cascas de umbu-caiá.

Os resultados da análise de correlação descritos na tabela 9 revelam que houve uma relação forte e direta entre os atributos avaliados pelos provadores, sendo que um influenciou no outro positivamente. Os atributos aparência e textura apresentaram a maior intensidade de correlação.

Tabela 8 - Teste de Correlação Linear de Pearson dos fishburgers

\begin{tabular}{ccccc}
\hline & APARÊNCIA & AROMA & SABOR & TEXTURA \\
\hline APARÊNCIA & 1 & $0,407\left(^{* *}\right)$ & $0,243\left(^{*}\right)$ & $0,618\left({ }^{* *}\right)$ \\
AROMA & 1 & $0,202\left(^{*}\right)$ & $0,264\left(^{* *}\right)$ \\
SABOR & & 1 & $0,359\left(^{* *}\right)$ \\
TEXTURA & & & 1 \\
\hline ** Correlação significativa ao nível de $0.01 /$ * Correlação significativa ao nível de 0.05
\end{tabular}

\section{Caracterização Físico-química dos Produtos Elaborados}

As análises físico-químicas foram desenvolvidas com as formulações de fishburgers adicionadas de farinha das cascas de umbu-cajá (FFC e EFC), as quais foram preferidas pelos provadores.

Com base nos resultados obtidos na análise do FFC e do EFC (Tabela 14 e 15), verifica-se que o experimento foi conduzido com muita precisão, demonstrando confiabilidade nos resultados. 
Tabela 9. Resultado da análise físico-química da formulação de Fishburger com Farinha de Cascas de Umbu-cajá

\begin{tabular}{cccc}
\hline & Média & D.P.* & C.V. $^{* *}$ \\
\hline Umidade (\%) & 73,37 & 0,62 & 0,84 \\
Cinzas Totais (\%) & 2,34 & 0,20 & 8,55 \\
Lipídeos (\%) & 3,98 & 0,61 & 5,33 \\
pH & 5,58 & - & - \\
Aw & 0,94 & - & - \\
\hline
\end{tabular}

* Desvio Padrão / ** Coeficiente de Variação

No que diz respeito à legislação, não existe uma exigência com relação ao conteúdo de minerais em produtos do tipo hambúrguer, no entanto, o valor obtido na análise de cinzas totais está dentro do que consta na Tabela de Composição dos Alimentos (TACO, 2011), que fica em torno de 2,9g/100g para hambúrgueres de bovino cru.

O Regulamento Técnico de Identidade e Qualidade (RTIQ) também não estabelece um percentual de umidade para o produto analisado, entretanto, sabe-se que a umidade de um alimento está relacionada com sua estabilidade, qualidade e composição, sendo a quantidade de água importante no processamento de vários produtos (CECCHI, 1999). O teor de umidade obtido nos fishburgers analisados (Tabela 14) assemelha-se ao encontrado por Carvalho (2009) em fishburguers de tilápia formulados com diferentes concentrações de farinha de trigo.

Outro resultado obtido nas análises químicas foi o percentual de gordura a partir da análise de lipídios; A gordura é um importante ingrediente no processamento cárneo, no qual é responsável por características relacionadas ao "flavor" em muitos produtos e também representa um fator relevante nas reações e alterações oxidativas. $\mathrm{O}$ valor obtido no produto analisado apresenta-se em conformidade com os padrões estabelecidos pelo Regulamento Técnico de Identidade e Qualidade para hamburguers, que permite até 23\% de lipídeos (BRASIL, 2000).

Os resultados de $\mathrm{pH}$ apresentados, encontram-se abaixo da neutralidade indicando maior estabilidade com relação à deterioração, o que se deve possivelmente à presença da farinha de cascas de umbu-cajá na formulação que é um produto ácido.

Levando-se em conta o fato de que a atividade de água (Aw) expressa o teor de água disponível para o crescimento microbiano e reações que possam deteriorar um alimento, os fishburgers analisados podem ser susceptíveis ao crescimento microbiano, portanto, é importante que sejam mantidos em refrigeração e manipulados em condições higiênico-sanitárias adequadas. 


\title{
4 Conclusão
}

O fishburger formulado apresenta-se dentro dos padrões básicos de qualidade estabelecidos pela legislação e a adição das farinhas de cascas de umbu e de umbu-cajá nestes produtos não interferiu a aceitação pelos provadores, indicando a possibilidade de uso como antioxidante natural para controlar o problema da oxidação lipídica em alguns alimentos.

\begin{abstract}
Products made with fish meat, as well other meat products, have low of lipids stability due to the liberation of enzymes in the meat disintegrated, incorporation of oxygen and presence of unsaturated fatty acid, which interfere the quality and validity of these products. The use of antioxidants has been considered a technique effective to retard or inhibit the oxidation of lipids in foods. Nevertheless, many studies have shown that some synthetic antioxidants can promote mutagenic and carcinogenic effects, among others, and for this reason, the search by natural antioxidants for replace or reduce the use of antioxidants synthetic, have been encouraged. Recent research shows that compounds existing in the fruit peel of umbuzeiro and related species, such as ascorbic acid, carotenoids and phenolic compounds have significant antioxidant potential. These findings led to the development of this work, for to evaluate the acceptance of formulations of fishburgers of Tilapia (Oreochromis niloticus), containing flour umbu peels (Spondias tuberosa) and umbu-caja (Spondias sp), as natural antioxidant. Were performed analyses Physico-chemical properties of flours, affective tests of acceptability of the product ready and statistical analysis of the variables. The results confirmed the suitability of products formulated with standards of identity and quality required and revealed that the addition of flours had no effect on acceptance by the panelists.
\end{abstract}

Key-words: phytochemicals; formulations; oxidation.

\section{Referências Bibliográficas}

BALASUNDRAN, M.; SUNDRAM, K.; SAMMAN. S. Phenolic Compounds in plants and agri-industrial by-products: Antioxidants Activity, occurrence, and potencial uses. Food Chemistry, v.99, p.191-203, 2006.

BOCHI, V. C. Otimização de uma formulação de fishburger de jundiá (Rhamdia quelen) visando o aproveitamento de resíduos da filetagem e do processamento de frutas. 2007. 99f. Dissertação (Mestrado em Ciência e Tecnologia de Alimentos) - Universidade Federal de Santa Maria, Santa Maria, 2007.

BRASIL. Agência Nacional de Vigilância Sanitária. Decreto no 50.040 de 24 de Janeiro de 1961. Normas Técnicas Reguladoras do Emprego de Aditivos Químicos em Alimentos. Diário Oficial da Republica Federativa do Brasil, 1961.

BRASIL. Ministério da Agricultura e do Abastecimento. Instrução Normativa $\mathbf{n}^{\circ}$ 20, de 31/07/00. Regulamentos técnicos de identidade e qualidade de almôndegas, fiambre, hambúrguer, kibe, presunto cozido e presunto. Brasília: Ministério da Agricultura e do Abastecimento, 2001.

BRASIL. Ministério da Agricultura e do Abastecimento. Regulamento técnico de identidade e qualidade de hambúrguer. Instrução Normativa n ${ }^{\circ}$ 20, de 31/07/2000. Diário Oficial da República Federativa do Brasil, Brasília, p. 7-9, 2000.

CARVALHO FILHO, D.U. Avaliação da qualidade de fishburger de tilápia (Oreochromis sp) em diferentes concentrações de farinha de trigo. 26f. Dissertação (Mestrado em Ciência Animal) - Universidade Federal do Piauí, 2009 . 
CARVAlHO, R.; LEMOS, D. Aquicultura e consumo de carnes no Brasil e no Mundo. Revista Panorama da Aquicultura, Rio de Janeiro, v.19, n.112, 2009.

CECCHI, H.M. Fundamentos teóricos e práticos em análise de alimentos. Campinas-SP: Editora da Unicamp, 207p., 2003.

DIMITRIOS, B. Sources of natural phenolics antioxidants. Trends in Food Science \& Technology, v. 17, n. 9, 2006.

DURAN, R. M. e PADILLA, R. B. Actividad antioxidante de los compuestos fenolicos. Grassas y Aceites, v.44, n. 2, 1993.

FOOD AGRICULTURE ORGANIZATION OF THE UNITED NATIONS (FAO), 2011. Disponível em: http://www.fao.org/figis/servlet/SQServlet?file=/usr/local/tomcat/FI/5.5.23/figis/webapps/figis/temp/hqp_4090.xml\&ou ttype $=$ html Acesso em: 08 out. 2012.

FOOD AGRICULTURE ORGANIZATION OF THE UNITED NATIONS (FAO), 2007. Disponível em: http://www.fao.org/fishery/en Acesso em: 17 nov. 2011

GELSLEICHTER, D. A. Avaliacao da atividade antioxidante e quantificacao dos principais constituintes bioativos de algumas variedades de frutas citricas. Tese (Doutorado) - Universidade Estadual Paulista,Araraquara, p.19-24. 2009,

HSU, C. K.; CHIANG, B. H. Effects of water, oil, starch, calcium carbonate and titanium dioxide on the colour and texture of threadfin and hairtail surimi gels. International Journal of Food Science \& Technology, Oxford, v. 37, n. 4, p. 387-393, 2002.

HUSSAIN, M.G. Farming of Tilapia. Breeding Plans, Mass Seed Production and Aquaculture Techniques. 149p., 2004.

JACZYNSKI, J. Proteins, lipid recovered from fish-processing by-products. Global Aquaculture Advocate, St. Louis, v. 8, n. 2, p. 34-36, 2005.

JORGE N., et.alli. Composição centesimal e atividade antioxidante do extrato de sementes de maracujá (passiflora edulis) em óleo de soja. Pesquisa Agropecuária Tropical, v. 39, n. 4, p.380- 385, 2009.

KUBITZA, F. Tilápia: tecnologia e planejamento na produção comercial. Jundiaí: Acqua \& Imagem, 289 p., 2003. LUTZ, Instituto Adolf. Normas Analíticas do Instituto Adolf Lutz: Métodos Químicos para Análise de Alimentos. 3 ed. São Paulo, 1985.

LUZIA, D. M. M.; JORGE N. Potencial antioxidante de extratos de sementes de limao (Citrus limon). Ciência e Tecnologia de Alimentos, Campinas, v. 30, n. 2, p. 489-493, 2010.

MELO, E. A. e ANDRADE, R. A. M. S. Compostos bioativos e potencial antioxidante de frutos do umbuzeiro. Alimentos e Nutrição, Araraquara, v. 21, n. 3, p.453-457, 2010.

MINISTÉRIO DA PESCA E AQUICULTURA. O potencial brasileiro para a aquicultura. 2010. Disponível em: http://www.mpa.gov.br/\#aquicultura/informacoes/potencialbrasileiro. Acesso em: 08 out. 2011.

MOREIRA, A.C.C.G. Caracterização de frutos de genótipos de cajá-umbuzeiras: teor de fitoquímicos bioativos e potencial antioxidante. 122f, Dissertacao (Mestrado em Ciência e tecnologia de Alimentos) - Departamento de Ciencias Domesticas, Universidade Federal Rural de Pernambuco, Recife, 2011.

OETTERER, M.; REGITANO D’ARCE, M.A.; SPOTO, M.H.F. Fundamentos de ciência e tecnologia de alimentos, São Paulo: Manole, 611p, 2006.

OLIVEIRA, C. S.; ALVES, F. S. Educação nutricional em unidade de alimentação e nutrição, direcionada para consumo de pratos protéicos: um estudo de caso. Alimentos e Nutrição. v.19, p.435-440, out./dez. 2009.

OSTRENSKY, A.; BOEGER, W.A.; CHAMMAS, M.A. Potencial para o desenvolvimento da aquicultura no Brasil. In: Aquicultura no Brasil O DESAFIO É CRESCER. 160p., 2008. 
PARMigiAnI, P., TORRES, R. A caminho da elite do agronegócio. Revista Aquicultura e Pesca. Edição 10, p.26-34, 2005.

RAMALHO, V. C. e JORGE, N. Antioxidantes utilizados em óleos, gorduras e alimentos gordurosos. Química Nova, Sao Paulo, v. 29, n.4, p.755-760, 2006.

SANTOS, M. B.; CARDOSO, R.L.; FONSECA, A.A.O.; CONCEIÇÃO, M.N. Caracterização e qualidade de frutos de umbu-cajá (spondias tuberosa x s. Mombin) provenientes do recôncavo sul da Bahia. Revista Brasileira de Fruticultura, Jaboticabal, v. 32, n. 4, p. 1089-1097, 2010.

SARY, C.; FRANCISCO, J.G.P.; DALLABONA, B.R. Influência da lavagem da carne mecanicamente separada de tilápia sobre a composição e aceitação de seus produtos. Revista Acadêmica de Ciências Agrárias e Ambientais, Curitiba, v. 7, n. 4, p. 423-432, out/dez. 2009.

SHUI, A. G.; LEONG, L. P. Residue from star frit as valuable source for functional food ingredients and antioxidant nutraceuticals. Food Chemistry, v.97, p.277-284, 2005.

SILVA, R.C.O.; NASCIMENTO, J. D. M. ; CURVELO, A. C. ; ARAUJO, C. R. ; LIMA, V.L.G. de ; MACIEL, M.I.S ; SILVA, C. G. M. ; MELO, E. A. Atividade antioxidante de extratos das cascas desidratadas de frutos de cajá umbuzeiros. Jornada de Ensino, Pesquisa e Extensão - JEPEX. Universidade Federal Rural de Pernambuco, Recife, 2010 .

SIMÕES, M.R.; RIBEIRO, C.F.A.; RIBEIRO, S.C.A.; MURR, F.E.X. Composição físico-química, microbiológica e rendimento do filé de tilápia tailandesa (Oreochromis niloticus). Ciência e Tecnologia Alimentar, v. 27, n. 3, p. 608$613,2007$.

SOARES, M.; WELTER, L.; KUSKOSKI, E.M.; GONZAGA, L.; FETT, R. Compostos fenólicos e atividade antioxidante da casca de uvas niagara e Isabel. Revista Brasileira de Fruticultura, Jaboticabal, v.30, n.1, p.059-064, 2008 .

SUMMO, C.; CAPONIO, F.; PASQUALONE, A. Effect of vacuum packaging storage on the quality level of ripened sausages. Meat Science, v.74, p. 249-254, 2006.

TACO - Tabela brasileira de composição de alimentos / NEPA - UNICAMP.- 4. ed. rev. e ampl. Campinas: NEPAUNICAMP, 2011. $161 \mathrm{p}$.

TENUTA-FILHO, A.; JESUS, R. S. Aspectos da utilização de carne mecanicamente separada de pescado como matéria-prima industrial. Boletim da Sociedade Brasileira de Ciência e Tecnologia de Alimentos, v.37, p.59-64, 2003.

TOKUR, B.; POLAT, A.; BEKLEVIK, G.; ÖZKÜTÜK, S. Changes in the quality of fishburger produced from tilapia (Oreochromis niloticus) during frozen storage $\left(18^{\circ} \mathrm{C}\right)$. European Food Research and Technology, Berlin, v. 218 , n. 4 , p. 420-423, 2004.

WEBER, J. Estabilidade Lipídica de Filés de Jundiá (Rhamdia quelen). 81f., Dissertação (Mestrado em Ciência e Tecnologia dos Alimentos) - Universidade Federal de Santa Maria-RS, 2007.

Submetido em 19 jun. 2013, Aceito para publicação em 23 jun. 2014, Publicado em 28 dez. 2014. 